\title{
Improved and Energy Efficient Olsr Protocol Using Spanning Tree in Manet
}

\author{
Priyanka Patil ${ }^{1}$, M.A.Rizvi ${ }^{2}$ \\ ${ }^{1}$ (Student, M.Tech, Department of Computer Engineering and Applications, NITTTR Bhopal, India) \\ ${ }^{2}$ (Associate Professor, Department of Computer Engineering and Applications, NITTTR Bhopal, India)
}

\begin{abstract}
Mobile Adhoc Network is an autonomous and decentralized network. Its topology changes dynamically and message overhead is more due to its frequent change of topology in network. For reliable transmission there is need to design a network which overcomes these problems like; overhead, Link breakage, power consumption etc. In this paper an attempt is made to overcome above mentioned issues by devising a new concept by modifying existing standard OLSR protocol using spanning tree in network. This approach has improved Packet Delivery Ratio and Throughput also uses minimum energy while transmitting.
\end{abstract}

Keywords: EOLSR (Efficient Optimized Link State Routing), MANET, Multicast, OLSR, Overhead, Spanning Tree.

\section{Introduction}

Mobile Adhoc Network is self established network. It is a decentralized network in which mobile nodes are connected together via wireless links. MANET infrastructure less network and can be deployed quickly, without having any prior planning or construction. MANET contains number of mobile nodes these nodes act as host as well as router in the network. Each node in the network has identical features, responsibilities and capabilities and hence it forms a completely symmetric environment. Communication among nodes is established on the bases of data packet transmission via wireless route formed between source and destination nodes of the network [1][2]. MANET has various applications in field where rapid deployment of a MANET is required like Military Battlefield, Commercial Sector like disaster rescue operations, Personal Area Network (PAN), Local Level etc. MANET has number of challenges which affect the performance of network. Mobility, link breakage, reliability, security and battery power are some of issues which are faces by network and its performance. In this paper an approached is used to deal with challenge of Link Breakage, Mobility, overhead and scalability along with power consumption. In this paper an attempt is made to overcome above mentioned issues by devising a new concept by modifying existing standard OLSR protocol using spanning tree in network. This approach has improved Packet Delivery Ratio and Throughput also uses minimum energy while transmitting.

\section{Realated Work}

An analysis is done on different forms of spanning tree-based topologies that could be used for efficient broadcasting in mobile ad hoc networks (MANETs) [3]. They consider three topologies minimum distance, predicted link expiration time and the minimum velocity broadcast topology for different condition of network density and node mobility. A spanning tree based broadcast topology exists at a particular time instant if all the edges of the tree exist in the underlying network graph corresponding to the snapshot captured at that time instant. It repeats the process if currently known spanning tree is not exist. Link expiration time Broadcast topology (LET-BT) based spanning tree is more stable than these trees had a larger value for the edge distance ratio as well as had a larger diameter. The Minimum Velocity based Broadcast Topology (MV-BT) trees also had an edge distance ratio (similar to that of the LET-BT); but they were not stable. On the other hand, the MVBT based spanning trees incurred the least diameter (a measure of the delay incurred in receiving a broadcast message at all the nodes in the network) among the three topologies.

A great work is done in number of studies on spanning tree related multicast route which includes disjoint route using minimum spanning tree, increase connectivity index with bandwidth constraint and survivability with respect to multicast route failure tolerance in K-connected MANET[4][5][6]. For disjoint route it uses prism's spanning tree algorithm in that they discusses route tolerance multicasting using spanning tree [4]. In cost of edge is considered as connectivity index of the vertices of graph [5]. With increasing spanning tree in the network connectivity also increases. It is significant to study connectivity, route failure tolerance and energy of the network to optimize the design of the multicast routing protocol by means of cross layer interaction across the layers of the network. If all (K-1) routes fail then it has an alternate route Kconnected route for transmission. The algorithms are devised and coded to compute number of spanning trees as well as Edge Disjoint Minimum Spanning Trees in K-connected networks [6]. 


\section{Methodology}

MANET deals with Number of limitation such as power consumption, Link Breakage, overhead, dynamic topology changes etc. To deal with these challenges Network will be design using spanning tree. Spanning tree has the property of providing loop free network. By applying spanning tree in MANET and it will help the network for reliable connectivity and rate of packet receiving will be increased. To achieve the goal standard OLSR is used along with the spanning tree. For verifying the mentioned concept standard OLSR is modified as per the concept using spanning tree for more reliable connectivity and increased throughput and reduced power consumption. Discrete Event Based Network Simulator is used for simulating standard OLSR and modified OLSR which will known as Efficient OLSR (EOLSR) as the output of this dissertation. Spanning tree is a graph which is connected to all the vertices of the graph but it does not form cyclic network [7]. For establishing connection in the network it has following steps:

1. Firstly consider a node as a MPR node with respect to MPR node it calculate distance with other nodes.

2. For calculating distances to each node it uses Euclidean formula with respect to MPR node

$\sqrt{\left(\left(\mathrm{x}_{\mathrm{n}}{ }^{2}-\mathrm{x}_{\mathrm{m}}{ }^{2}\right)-\left(\mathrm{y}_{\mathrm{n}}{ }^{2}-\mathrm{y}_{\mathrm{m}}{ }^{2}\right)\right)}$. The process of communication is started in the network by HELLO message of OLSR protocol. On the bases of calculated distances connect the path in increasing order of the distances. As a result we got a spanning tree network where every node is connected with two other nodes and store the information of these two nodes only.

3. MPR node is considered as first node and IP addresses will be assigned to nodes in the network. Connect each node with the ascending order of IP addresses.

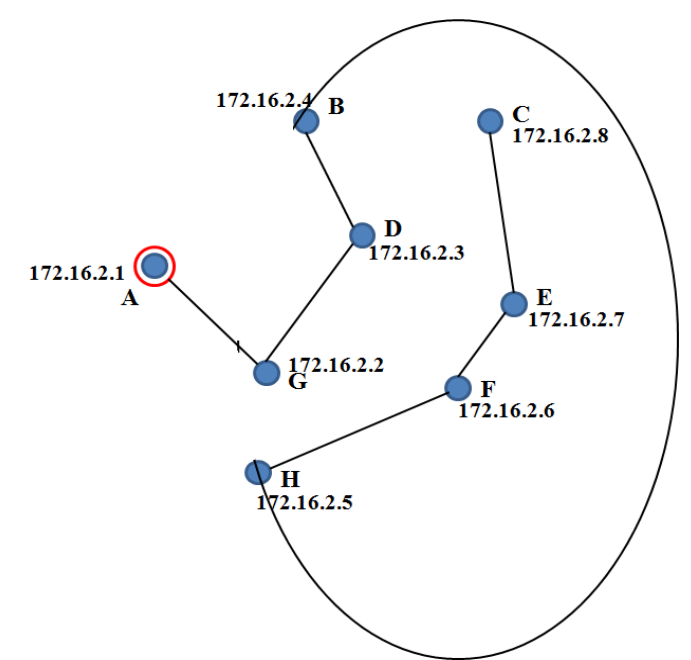

Fig. 1: Spanning tree based connected network [8]

IV. Simulation

Network simulator OPNET MODELER 14.0 is used for designing the network and analyzing performances of Packet Delivery Ratio and Throughput. Two networks are designed with 25 and 50 nodes on the bases of Table 1:

\begin{tabular}{|c|c|}
\hline Simulation Time & 300 seconds \\
\hline Protocol & OLSR and EOLSR (Developed) \\
\hline Area: & $\mathbf{1 0 0 0} \times 1500$ meter \\
\hline Traffic & CBR \\
\hline Channel & Wireless \\
\hline Operation mode & Random waypoint \\
\hline Mobility & Omni directional \\
\hline Antenna & $\mathbf{5 0}$ \\
\hline IFQ & $\mathbf{2 5}$ and 50 \\
\hline Nodes &
\end{tabular}

Table 1: Parameters for Designing Network 


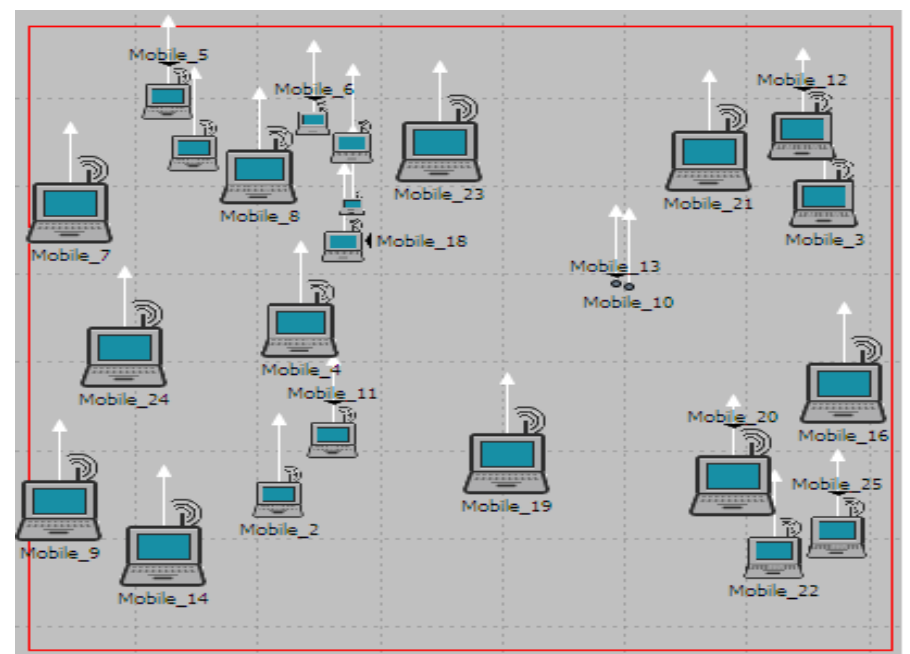

Fig. 2: Scenario with 25 nodes in the network

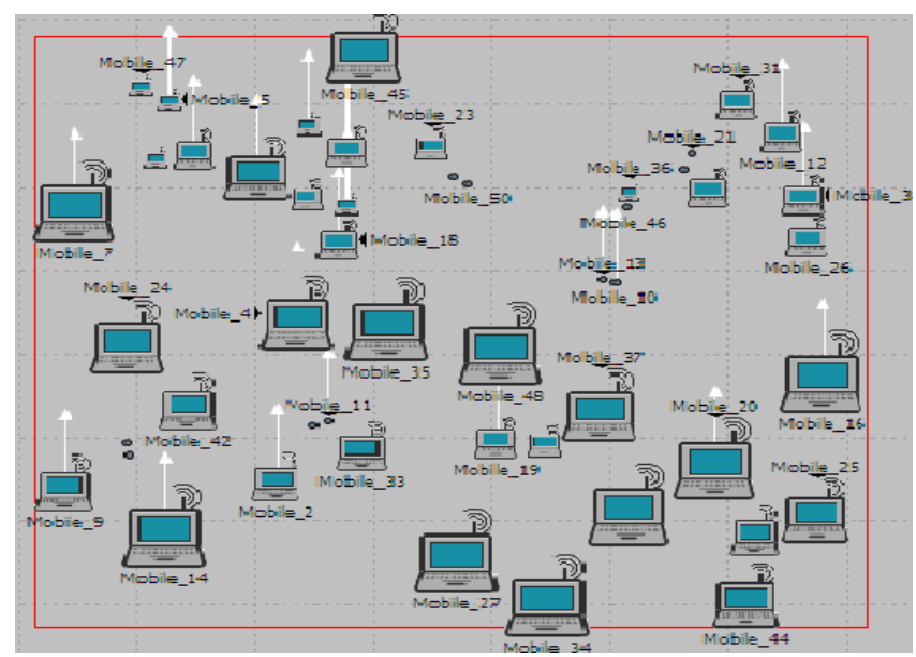

Fig. 3: Scenario with 50 nodes in the network

\section{Results}

Packet Delivery Ratio:

PDR is a parameter which is used for analysis the performance of algorithms in network. It is the ratio of successful packet received at destination to the packet send from source. A high packet delivery ratio is desired in a network. Greater value of PDR gives better performance.

\section{PDR $=\sum$ Number of Packet Receive $\sum$ Number of Packet Send}

\begin{tabular}{|c|c|c|c|c|c|c|}
\hline \multirow{2}{*}{25 Nodes } & OLSR & 41.01 & 42.55 & 47.56 & 41.98 & 45.37 \\
\cline { 2 - 7 } & EOLSR & 42.60 & 44.64 & 49.49 & 43.03 & 47.27 \\
\hline \multirow{5}{50}{ Nodes } & OLSR & 41.00 & 42.30 & 47.70 & 41.10 & 45.98 \\
\cline { 2 - 7 } & EOLSR & 42.70 & 44.74 & 49.60 & 43.10 & 47.01 \\
\hline \multicolumn{2}{|c|}{ Speed } & $3 \mathrm{~m} / \mathrm{s}$ & $6 \mathrm{~m} / \mathrm{s}$ & $9 \mathrm{~m} / \mathrm{s}$ & $12 \mathrm{~m} / \mathrm{s}$ & $15 \mathrm{~m} / \mathrm{s}$ \\
\hline
\end{tabular}

Table 2: PDR for 25 and 50 nodes Scenarios

The data also reveals that data was collected on five different speeds $3 \mathrm{~m} / \mathrm{s}, 6 \mathrm{~m} / \mathrm{s}, 9 \mathrm{~m} / \mathrm{s}, 12 \mathrm{~m} / \mathrm{s}$ and 15 $\mathrm{m} / \mathrm{s}$ using Network Simulator. After collecting the data further analysis is shown with Graph 1 and Graph 2 for PDR with 25 and 50 nodes respectively. 


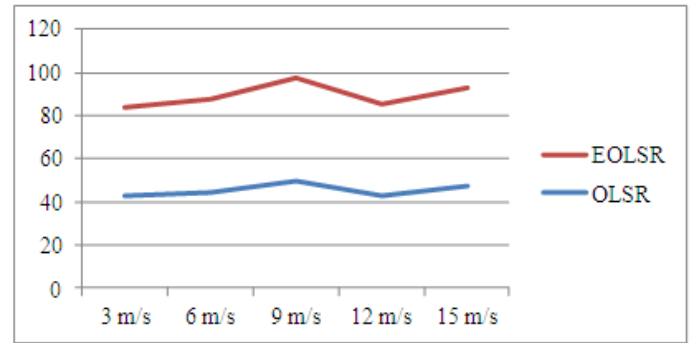

Graph 1: Improvement in Packet Delivery Ratio with 25 nodes

Graph 1 shows the performance of algorithms at five different speeds. OLSR and EOLSR at different speeds have been compared. The graph of 25 nodes Scenario between OLSR and EOLSR shows better performance than OLSR algorithm on PDR parameter. It means that EOLSR is more reliable than OLSR in terms packet delivery.

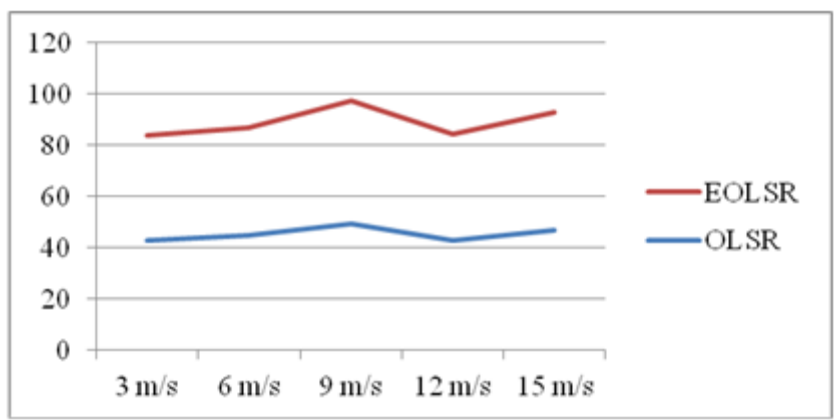

Graph 2: Improvement in Packet Delivery Ration with 50 nodes

Graph 2 is drawn on the bases of 50 nodes Scenario. Graph 2 shows improvement in PDR with 50 nodes. So it can be summarize that the EOLSR with 50 nodes is also performing better that OLSR hence more reliable.

\section{Throughput:}

Throughput is defined as; the ratio of the total amount of data that reaches a receiver from the sender to the time it takes by the receiver to receive the last message is called as throughput. Throughput is expressed as bytes or bits per sec (byte/sec or bit/sec). It is total number of delivered packets to time taken by last packet for delivery.

\section{Throughput $=$ Number of Delivered Packet}

Transfer Time

If a protocol shows high throughput so it is the efficient and best protocol than the routing protocol which have low throughput. Higher throughput gives better performance in the network. In Table 2 results of simulation is collected.

\begin{tabular}{|c|l|c|c|c|c|c|}
\hline \multirow{2}{*}{25 Nodes } & OLSR & 4555 & 4678 & 4598 & 4557 & 4577 \\
\cline { 2 - 7 } & EOLSR & 4677 & 4798 & 4697 & 4600 & 4627 \\
\hline \multirow{2}{*}{50 Nodes } & OLSR & 4550 & 4600 & 4600 & 4580 & 4597 \\
\cline { 2 - 7 } & EOLSR & 4670 & 4801 & 4777 & 4690 & 4703 \\
\hline \multicolumn{2}{|c|}{ Speed } & $3 \mathrm{~m} / \mathrm{s}$ & $6 \mathrm{~m} / \mathrm{s}$ & $9 \mathrm{~m} / \mathrm{s}$ & $12 \mathrm{~m} / \mathrm{s}$ & $15 \mathrm{~m} / \mathrm{s}$ \\
\hline
\end{tabular}

Table 3 Throughput with 25 and 50 nodes network

For the parameter Throughput a Simulation has been done on two scenarios of network 25 and 50 nodes on standard OLSR and Improved EOLSR the data is shown in Table 3.and further analysis is done with Graph 3 and Graph 4 for Throughput with 25 and 50 nodes respectively. 


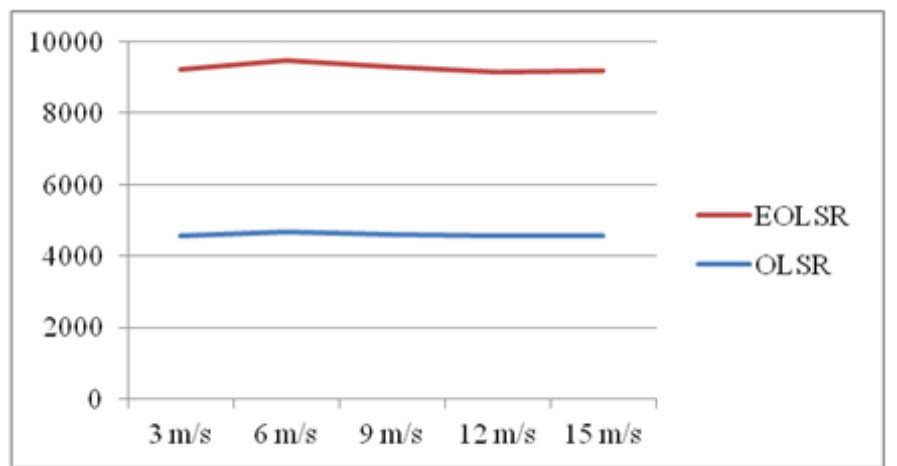

Graph 3: Improvement in Throughput with 25 nodes

Graph 3 is the graphical representation of throughput of 25 nodes network. This graph shows the performance of OLSR and EOLSR with 25 nodes network. An analysis can be drawn that EOLSR provides efficient throughput than standard OLSR algorithm.

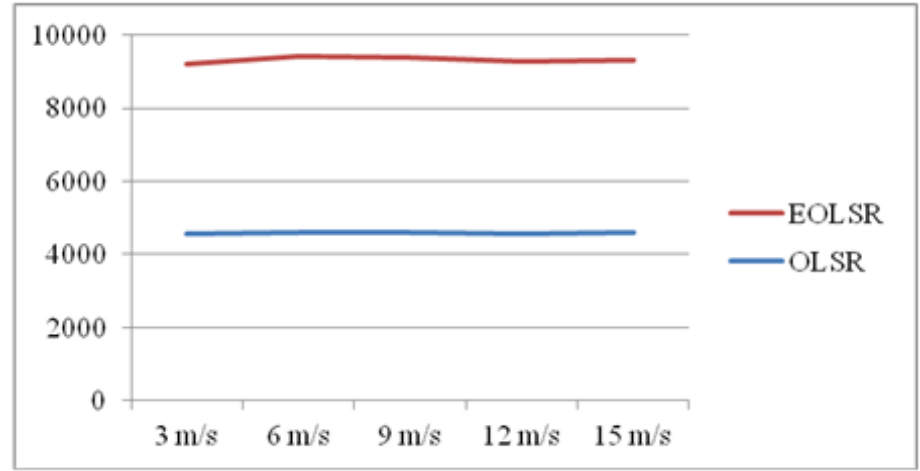

Graph 4: Improvements in Throughput with 50 Nodes

Both the Graphs 3 and 4 of throughput with 25 and 50 nodes shows that the performance of EOLSR is better in case of throughput. On the bases of analyses it observes that EOLSR has better throughput for dense network as compare to lesser dense network like 25 nodes.

\section{Conclusion}

MANET has several issues and challenges as discussed above, so need is felt that new algorithm should designed which will address these issues. In this paper an attempt is made to overcome above mentioned issues by devising a new concept by modifying existing standard OLSR protocol using spanning tree in network. This approach has improved Packet Delivery Ratio and Throughput also uses minimum energy while transmitting. The newly designed EOLSR algorithm was compared with the standard OLSR using OPNET simulator and found the EOLSR is better performing. Though this concept has to be tested on other parameters of network like end-to-end delay, jitter and on high speed transmission.

[1]. Mobile Ad Hoc Networks Asis Nasipuri

\section{References}

[2]. http://www.eexploria.com/manet-mobile-ad-hoc-network-characteristics-and-features/

[3]. Arun Kumar B. R, Lokanatha C. Reddy and Rajan and Prakash S. Hiremath "Route Failure Tolerant Multicast In Mobile Ad Hoc Networks Using Disjoint Minimum Spanning Trees" second IEEE ICCSIT 2009, August 8 - 11, 2009, Beijing, China

[4]. Arun Kumar B. R, Lokanatha C. Reddy and Rajan and Prakash S. Hiremath "Route Failure Tolerant Multicast in Mobile Ad Hoc Networks Using Disjoint Minimum Spanning TreesBased on Connectivity Index with Bandwidth constraint” 2010 IEEE 2nd International Advance Computing Conference

[5]. Arun Kumar B. R, Lokanatha C. Reddy and Rajan and Prakash S. Hiremath "Restoring the Connectivity in K-Connected MANET When All Edge Disjoint Minimum Spanning Trees Fail” IJCA Special Issue on "Mobile Ad-hoc Networks" MANETs, 2010

[6]. Abhishek Patil, A-H. Esfahanian, Li Xiao and Yunhao Liu "Resource Allocation using Multiple Edge- Sharing Multicast Trees"

[7]. Priyanka Patil, Monika Sahu, and M.A.Rizvi "Proficient Data Communication in MANET using Spanning Tree" $5^{\text {th }}$ International Conference on Computational Intelligence and Communication Network (CICN) 2013 27-29 ${ }^{\text {th }}$ September 2013. 